\title{
Einige Gedanken zur Sentenz: „... aber wir wissen nicht, warum Homöopathie wirkt"
}

\author{
Wolfgang Würger
}

\section{ZUSAMMENFASSUNG}

Das Ziel eines wissenschaftlichen Paradigmas in der Situation der Paradigmenkonkurrenz besteht nicht zuletzt darin, das Selbstvertrauen seiner Gegner systematisch zu schwächen. So verfährt auch die Schulmedizin gegenüber der Homöopathie, indem sie ihr unablässig Defizite an Wissenschaftlichkeit zuzuschreiben versucht. Fatal ist, dass diese Vorwürfe von manchen Homöopathen - entgegen aller wissenschaftstheoretischen Evidenz-noch immer geglaubt werden. Diesen Zustand gilt es wissenschaftstheoretisch zu kritisieren und durch praktisches Handeln aufzulösen.

Schlüsselwörter

Wissenschaftlichkeit, Paradigma, Wirksamkeit,

Wissenschaftstheorie, Wissenschaftspolitik.

\section{SUMMARY}

The influence of a scientific paradigm in prevailing concurrence of paradigms depends not at least on its ability to weaken the self-confidence of its opponents. Conventional medicine is still very successful in its continual attempts to reproach homoeopathy with a lack of scientific status. It is surprising that some homoeopaths still are prepared to believe this although this reproach cannot offer any convincing scientific arguments and could therefore easily be repudiated. This inacceptable situation has to be changed both in theoretical as in practical respect.

Keywords

Scientific status, paradigm, efficacy, theory of science, politics

of science.

\section{Exposition der Problematik}

In den Diskussionen der letzten Jahrzehnte lautet der wiederkehrende Hauptvorwurf der Schulmedizin gegenüber der Homöopathie, dass der „wissenschaftliche Nachweis" einer Wirksamkeit unserer Medizin bis heute aussteht. Dabei wird zumeist gleichzeitig - sei es ausgesprochen oder unausgesprochen-unterstellt, dass ein solcher Wirksamkeitsnachweis auch kaum möglich sein kann, da die homöopathischen Arzneimittel über wenig oder gar keine Arzneisubstanz im materiellen Sinne verfügen. Träten dennoch therapeutische Wirkungen nach Anwendung homöopathischer Arzneien auf, dann müssten diese Wirkungen, so wird von der gegnerischen Seite aus postuliert, folgerichtig ausschließlich auf Placeboeffekte zurückgeführt werden.

Aber selbst wenn die Möglichkeit therapeutischer Wirksamkeit homöopathischer Arzneien von manchen Kritikern nicht vollständig in Abrede gestellt wird, so argumentieren viele von ihnen dahingehend, dass sich diese von den Homöopathen behaupteten therapeutischen Effekte nicht signifikant von Placebowirkungen abgrenzen ließen. In diesem Zusammenhang fordert die Schulmedizin üblicherweise von der Homöopathie die Erbringung eines Wirksamkeitsnachweises, und zwar gemäß den Vorstellungen von Wissenschaftlichkeit, die dem schulmedizinischen Paradigma selbst entnommen sind. Dabei werden immer wieder die randomisierten kontrollierten Studien ins Gespräch gebracht.

\section{Wissenschaftlich belegte Wirksamkeit}

Die Vertreter der Homöopathie hatten sich in der Vergangenheit als Antwort auf diese nicht abreißenden Vorhaltungen sehr bemüht gezeigt, den Anforderungen so gut wie nur möglich nachzukommen, um damit den Einwand fehlender Wirksamkeit unserer Arzneien zu entkräften bzw. zu widerlegen. In zahlreichen Studien konnte mittlerweile der Nachweis geführt werden, dass mit den homöopathischen Arzneien ein therapeutischer Nutzen verbunden ist, welcher sich überzeugend von Placeboeffekten unterscheiden lässt, und das auch in einer Weise, die methodisch durch die konventionelle Medizin nicht mit guten Gründen beanstandet werden kann. Die Wirksamkeit der Homöopathie darf insofern heute, auch aus der Perspektive skeptischer Betrachter, als ausreichend wissenschaftlich belegt gelten.

\section{Wirksamkeit ohne naturwissenschaftlich nachgewiesene Arzneisubstanz}

Doch selbst dann, wenn die konventionelle Medizin diese Nachweise, die durch Studien und sich daran anschließende Metaanalysen geliefert werden, zu akzeptieren bereit wäre, bleibt aus ihrer Sicht noch immer eine offene Frage übrig, nämlich diejenige nach einer ihr ausreichend erscheinenden Begründung für diese - wir setzen dies hier einmal voraus-positiv nachgewiesene Wirksamkeit. Und eben mit dieser zweiten Frage, der Frage, wie es möglich sein kann, dass Arzneien, in denen kaum oder gar keine chemisch-physikalisch nachweisbare Arzneisubstanz enthalten ist, medizinisch wirken 
können sollen, gelingt es der Schulmedizin bis auf den heutigen Tag stellenweise noch immer, die Homöopathie durch die Verweigerung der Anerkennung von „Wissenschaftlichkeit" (in einem von ihr definierten Sinne) auch weiterhin in der Defensive und zugleich in einer ihr gegenüber unterlegenen Diskursposition zu halten.

\section{Usurpierte Deutungshoheit der Schulmedizin}

Die Schulmedizin beansprucht hierbei für sich die Autorität einer absoluten Schiedsgerichtsinstanz in Sachen Wissenschaftlichkeit von Medizin überhaupt. Werden von Teilnehmern wissenschaftlicher Diskurse solche universalen Ansprüche für das eigene Wissenschaftsmodell erhoben, wie die Schulmedizin es in unserem Falle oft noch tut, dann haben wir es mit polemischen Strategien im Rahmen der Paradigmenkonkurrenz zu tun, also mit Formen einer Auseinandersetzung, wo es in erster Linie um die Durchsetzung von Geltungs- und Machtansprüchen geht. Diesen gegenüber treten dann die Wahrheitskriterien, die in wissenschaftlicher Diskussion und Reflexion eigentlich höchste Priorität besitzen sollten, eher zurück. Strategien der genannten Art zielen letztlich darauf $a b$, die Homöopathie im Stande einer inferioren, weil angeblich wissenschaftlich nicht ausreichend begründeten Medizin zu halten. Dabei behauptet die Schulmedizin in diesem Prozess nicht nur, über das einzige wissenschaftlich korrekte Paradigma zu verfügen, sondern beansprucht zugleich in Sachen „Wissenschaftlichkeit“ die Deutungshoheit ausschließlich für sich.

\section{Angebliche Unwissenschaftlichkeit}

Selten genug - und da sind wir schon mittendrin in unserer Problematik - wird bemerkt, dass sich die Schulmedizin durch die Erhebung solcher absoluter, uneinlösbarer Geltungsansprüche in eklatanten Widerspruch zu den Resultaten der wissenschaftstheoretischen Diskussionen der letzten 100 Jahre stellt und dass sie sich eben dadurch selbst außerhalb des Rahmens moderner Wissenschaftlichkeitsdefinitionen platziert. Anstatt nun aber solche unhaltbaren Ansprüche, wie sie die Schulmedizin erhebt, mit guten wissenschaftstheoretischen Gründen zurückzuweisen, die lange schon bereitliegen, verstehen sich noch immer nicht wenige Homöopathen erstaunlicherweise dazu, den schulmedizinischen Vorwurf betreffs der angeblich ungenügenden wissenschaftlichen Basis der Homöopathie mehr oder weniger widerspruchslos und widerstandslos zu akzeptieren.

Dieser bedenklichen Haltung begegnen wir beispielsweise in einem Ausspruch, den man mit schöner Regelmäßigkeit immer wieder aus den Reihen der Homöopathie vernehmen kann und der da sinngemäß lautet: Wir, also die Homöopathen, wüssten zwar, dass Homöopathie wirkt, aber wir wüssten nicht, warum sie wirkt. Zwar beharrt man dabei auf der Überzeugung, dass die homöopathische Medizin wirksam sei, gesteht aber gleichzeitig zu, dass in der (angeblichen) Nichtbeantwortbarkeit der Warum-Frage ein zentrales wissenschaftliches Defizit besteht, wodurch die Homöopathie in Hinblick auf „Wissenschaftlichkeit“ im Vergleich mit der Schulmedizin im Hintertreffen liege und sich deren Wissenschaftsmodell vielleicht doch am besten zum Vorbild nehmen sollte.

\section{Fehlerhafte wissenschaftstheoretische Annahmen}

Homöopathen, die sich einen solchen Ausspruch zu eigen machen, beziehen damit-unabhängig davon, ob sie das wissen und wollen oder nicht-die folgenden wissenschaftstheoretischen Positionen. Gegenüber den Anwürfen der Schulmedizin konzedieren sie

- dass die Homöopathie wissenschaftliche Defizite aufweist, da sie das Zustandekommen ihrer Arzneiwirkungen nicht überzeugend gemäß der schulmedizinischen Vorstellungen von Wissenschaftlichkeit erklären kann,

- dass eine überzeugende Erklärung ihrer Arzneiwirkung überhaupt nur und nur dann vorliegt und vorliegen kann, wenn sie im Rahmen des nämlichen naturwissenschaftlich orientierten Paradigmas erbracht werden kann, dem sich die Schulmedizin verpflichtet fühlt,

- dass das naturwissenschaftlich orientierte Wissenschaftsmodell der Schulmedizin ganz zu Recht seine Universalitätsansprüche in Sachen (medizinischer) Wissenschaftlichkeit erhebt und dass es damit

- als einzig gültiger Maßstab an alle anderen Medizinen und somit auch an die Homöopathie angelegt werden kann.

Die noch immer kursierenden negativen Urteile der Schulmedizin über die angebliche Unwissenschaftlichkeit der homöopathischen Medizin, und darunter insbesondere der Hinweis, die Homöopathie wäre nicht in der Lage, in einer ausreichend wissenschaftlichen Beschreibung die Wirkungsweise ihrer Arzneien zu erklären, beruhen samt und sonders auf diesen genannten fehlerhaften wissenschaftstheoretischen Annahmen.

\section{Wissenschaftsposition der Homöopathie}

In diesem von homöopathischer Seite immer wieder zu hörenden Ausspruch, „wir wissen nicht, warum Homöopathie wirkt“, erscheinen mir gleich 2 Begriffe hochgradig problematisch und dringend klärungsbedürftig. Da ist zunächst die Frage, was in diesem Zusammenhang unter dem Begriff „Wissen“ verstanden werden soll, und daran anschließend, was bzw. wer mit dem Personalpronomen „wir“, dem Subjekt dieses Satzes, gemeint sein kann. Ich wende mich zunächst dem ersten Problembegriff zu, dem Begriff des „Wissens“. 
Erwartung von Wirksamkeit

Legen wir das Hahnemann'sche Wissenschaftsparadigma zugrunde, so dürften eigentlich keine Zweifel darüber auftauchen, warum unsere Arzneien, wenn sie lege artis ausgesucht und angewandt werden, wirksam sind. Diese Erwartung von Wirksamkeit können wir aus dem grundlegenden wissenschaftlichen Axiom der Homöopathie, dem Ähnlichkeitsprinzip, deduzieren, das heißt aus dem Wissen, dass eine Arznei, die gemäß der Symptomatik der Krankheit bestimmt wird, also die gleichen Symptomenqualitäten aufzuweisen hat wie diese, Heil- oder Linderungswirkungen hervorzubringen imstande ist und damit die, in den Worten Hahnemanns, „Verstimmung“ der Lebenskraft entweder beseitigen oder wenigstens vermindern kann.

\section{Warum?}

Um zu einer befriedigenden Beantwortung der WarumFrage zu gelangen - „Warum wirkt eine homöopathische Arznei?" -, ist es also erforderlich, dass wir auf das Wirkprinzip der Homöopathie rekurrieren und uns hierzu ihr vitalistisches Organismusmodell genauer ansehen. Der Organismus wird von Hahnemann als qualitativ bestimmbare Einheit angesehen, definiert über die Hypothese einer Lebenskraft, über die sämtliche Lebensprozesse ihre kohärente, geordnete und auf Gesundheit ausgerichtete Steuerung erfahren. Homöopathische Arzneien zielen von ihrem Ansatz her gemäß dem Wissenschaftsparadigma, innerhalb dessen sie zum Einsatz kommen, nicht primär auf die materielle Ebene des organischen Geschehens, welche man durch eine naturwissenschaftliche Beschreibung fasslich machen kann, sondern auf seine zentrale, übergeordnete Steuerungseinheit, wo alle Fäden zusammenlaufen, eben auf die Lebenskraft. Gelingt es nun, die Spezifität einer „Verstimmung“ der Lebenskraft, die sich in besonderen Krankheitsbildern zum Ausdruck bringt, durch eine dieser Spezifität entsprechende Arznei (Simile) aufzuheben, dann können wir die Wirksamkeit dieser Arznei am Patienten beobachten und die Gründe für diese Wirksamkeit in einer unserem Wissenschaftsparadigma zugehörigen Sprache beschreiben.

\section{Wirkung im Bereich der Lebenskraft}

Mit dem von manchen Homöopathen als problematisch empfundenen Begriff, dass der Charakter der homöopathischen Arzneien „geistartig“ sei, umschreibt Hahnemann genau das Faktum, dass diese Arzneien nicht primär auf materiell definierbaren Ebenen ihre Hauptwirkung entfalten, sondern wesentlich im Bereich der Lebenskraft, die wir nur als qualitative Einheit fassen können, im Vergleich zu der die allermeisten der quantifizierbaren Faktoren, auf die die Schulmedizin ihr Augenmerk richtet, nur von sekundärer Natur sind. Es ist diese Lebenskraft, die durch unsere Arzneien einen qualitativen Impuls erfährt und daraufhin zielgerichtet zu reagieren beginnt und die gewünschte Wirksamkeit entfaltet, die in der Wiederherstellung von Gesundheit besteht, zumindest jedoch in Schritten, die in diese Richtung weisen, selbst dann, wenn dies nur noch in palliativer Bescheidenheit möglich ist.

\section{Unzureichende naturwissenschaftliche Modelle}

Nimmt man diesen Sachverhalt kritisch genug wahr, dann kommt man kaum mehr um die Vermutung herum, dass es auch in wissenschaftlicher Hinsicht wenig weiterführend sein wird, wollte man diese qualitativen Prozesse, die im Organismus ablaufen und auf die die Homöopathie mit ihren hochspezifischen Arzneien einwirkt, im Rückgriff auf die quantifizierenden Modelle der Naturwissenschaften darzustellen versuchen. Selbst wenn man auf diesem Wege vielleicht manchmal zu nicht uninteressanten Einzelergebnissen gelangen könnte, wird man damit doch, allein schon bedingt durch den ontologisch unpassenden Charakter der angewandten Modelle, das Wesentliche dieses qualitativ zu definierenden Prozessgeschehens ganz zwangsläufig verfehlen.

\section{Paradigmendifferenz}

Die Wissenschaftssprache der Homöopathie ist somit, wie zu sehen, sehr wohl in der Lage, die Gründe für das Zustandekommen der Wirkung der homöopathischen Arzneien angemessen zu beschreiben und uns auf die Warum-Frage eine sehr präzise Antwort zu geben. Es handelt sich dabei, und niemand dürfte das überraschen, um die nämliche Antwort, die Hahnemann uns vor über 2 Jahrhunderten bereits gegeben hat. Dass die Schulmedizin mit dieser Antwort wenig anfangen kann, liegt auf der Hand, denn sie ist als Möglichkeit in ihrem Paradigma nicht vorgesehen. Stattdessen werden dort Auskünfte anderer Art erwartet, die nun hinwiederum mit dem Paradigma der homöopathischen Medizin keine Kongruenz aufweisen und ergo von ihr weder gegeben werden können noch gegeben werden müssen. Noch deutlicher lässt sich die Paradigmendifferenz in diesem Zusammenhang, eben im Zusammenhang der Diskussion der Begründung von Arzneiwirkungen, wohl kaum mehr artikulieren.

\section{Anerkennen des homöopathischen Wissenschaftsparadigmas}

Diese unüberbrückbare Differenz, die zwischen den Paradigmen der Schulmedizin und der Homöopathie besteht, ist längst bekannt, in erforderlicher wissenschaftlicher Breite wie Tiefe dargestellt und ausgeführt und bedürfte eigentlich keiner weiteren Erwähnung mehr, wenn nicht, aus einem erstaunlichen Impuls heraus, manche Homöopathen sich eben diesen Vorwurf der konventionellen Medizin-die Homöopathie könne nicht begründen, warum ihre Arzneien wirken-nach wie vor zu eigen machen wollen. Das aktuelle Problem innerhalb der Homöopathenschaft scheint m. E. also weniger darin zu liegen, dass wir keine befriedigende Antwort auf die 
Warum-Frage haben, sondern vielmehr darin, dass viele Homöopathen die Antwort, die uns Hahnemann gegeben hat, heute entweder nicht mehr verstehen können oder, aus welchen Gründen auch immer, nicht (mehr) verstehen wollen.

Die Voraussetzung für das Verstehen der Antwort, warum Homöopathie wirkt, liegt darin, dass man das homöopathische Wissenschaftsparadigma (Ähnlichkeitsprinzip, Hypothese einer Lebenskraft, Organismusmodell) in seiner Gesamtheit als gültig anzuerkennen bereit ist, und sei es auch nur im hypothetischen Sinne. Dafür, für die wissenschaftliche Verfasstheit unseres Paradigmas, wurden in der Vergangenheit bereits viele gute Gründe angeführt, die hier nicht wiederholt zu werden brauchen. Nur so viel: Das homöopathische Wirkungsmodell ist in theoretischer Hinsicht in sich logisch stringent, in praktischer Hinsicht empirischer Überprüfbarkeit zugänglich und liefert, wenn man sich in wissenschaftlicher Haltung auf seine paradigmatischen Grundlagen einlassen will, genügend Material und Hinweise, um eine kritische Reflexion auf seine Bewährtheit durchführen zu können, woraus sich dann auch überzeugend rekonstruieren lässt, warum die homöopathischen Arzneien wirken.

\section{Wissenschaftstheoretische Irritationen in der Homöopathie}

Den Vertretern des konkurrierenden Paradigmas der Schulmedizin kann man es gewiss nicht verargen, wenn sie auf der Gültigkeit ihres eigenen Wissenschaftsmodells bestehen und von dort her dem homöopathischen Paradigma die Anerkennung versagen. Überraschend hingegen erscheint es, wenn Homöopathen Probleme haben, das homöopathische Wissenschaftsparadigma anzuerkennen und sich im Rückgriff auf dasselbe die Wirksamkeitsfrage in begründeter Weise zu beantworten. Denjenigen Homöopathen, die erklären, sie wüssten nicht, warum Homöopathie wirkt, schwebt also offenbar eine ganz andere Art von wissenschaftlichem „Wissen“ vor, als dasjenige, das unsere Wissenschaft verkörpert und bereitzustellen vermag, nämlich eine Art von Wissen, das in seinen leitenden Grundsätzen schulmedizinischem Wissen verpflichtet ist und sich mit dessen Paradigma als kompatibel erweist.

Man darf vermuten, dass diese Homöopathen das Diktum der konventionellen Medizin - faktisch nichts anderes als ein Verdikt -, die Homöopathie sei in wissenschaftlicher Hinsicht defizitär, akzeptiert haben und mögliche Abhilfe dagegen in der Übernahme entweder des schulmedizinischen Wissenschaftsmodells insgesamt oder doch einiger seiner zentralen Bestandteile sehen. Es verwundert nicht, dass die Homöopathie, wenn sie diese Position übernimmt, besonders angreifbar ist, wenn von der Gegenseite wieder einmal die Warum-Frage nach der Wirksamkeit der homöopathischen Arzneien ins Feld geführt wird.

\section{Wissenschaftstheoretische Denkfehler}

Unabhängig davon, dass es einem jeden freisteht, sich für das eine oder das andere Wissenschaftsparadigma in der Medizin zu entscheiden, das man, aus welchen Gründen auch immer, bevorzugen möchte, unterliegen Homöopathen, die eine naturwissenschaftlich ausgerichtete Begründung für die Wirksamkeit homöopathischer Arzneien erwarten bzw. überhaupt nur für möglich halten, gleich mehreren-expliziten oder implizitengewichtigen wissenschaftstheoretischen Denkfehlern, die sie in der Regel aus dem schulmedizinischen Paradigma ohne weitere kritische Überprüfung ihrer Tauglichkeit und Anwendbarkeit für die Wissenschaftsgestalt der Homöopathie übernommen haben.

- Man geht davon aus, dass das Attribut „wissenschaftlich" denkungsgleich ist mit der Zuschreibung „naturwissenschaftlich“ und übersieht, dass es verschiedene Arten von Wissenschaften (hermeneutische, analytische, dialektische etc.) gibt, deren Wissenschaftsmodelle ihre jeweilige Berechtigung aus ihrer Gegenstandskonstitution beziehen.

- Man sitzt - teilweise oder ganz - dem in der Schulmedizin noch immer weit verbreiteten Selbstmissverständnis auf, die Medizin sei eine Naturwissenschaft, weshalb eine jede Medizin, die „wissenschaftlich“ sein wolle, diesem Wissenschaftsmodell nachzustreben habe, das angeblich die Schulmedizin vorexerziert.

- Man gesteht dem in der Schulmedizin applizierten naturwissenschaftlich orientierten Wissenschaftsmodell die von ihm erhobenen absoluten Geltungsansprüche in Sachen „Wissenschaftlichkeit“ zu. Diese Geltungsansprüche können allerdings, wie man weiß, samt und sonders keiner wissenschaftstheoretischen oder wissenschaftskritischen Prüfung auch nur im Entferntesten standhalten. Allein dogmatische, von ihren Absichten wie von ihren Auswirkungen her totalitäre Denksysteme neigen heute, im Zeitalter eines allgemein anerkannten Rationalitäts- und damit Wissenschaftspluralismus, noch dazu, solche hoffnungslos überzogenen Ansprüche zu erheben. Keine Wissenschaft, die sich selbst als solche versteht und die in der Wissenschaftsgemeinde unter diesem Namen anerkannt werden will, wird sich hierzu versteigen.

\section{Absolutheitsansprüche der Schulmedizin}

Nur wenn sie diese Positionen, in der sich die Schulmedizin in ideologischer Weise selbst als die einzig mögliche wissenschaftliche Medizin stilisiert, als gültig zu übernehmen bereit sind, können Homöopathen zu der fragwürdigen Einschätzung gelangen, wir wüssten nicht, warum unsere Arzneien wirken, da eine naturwissenschaftlich 
einigermaßen befriedigende Beschreibung bis heute nicht vorliegt und - was ausgesprochen vorteilhaft ist für die Position der Kritiker der Homöopathie - mit sehr groBer Wahrscheinlichkeit auch niemals wird vorliegen können.

Diese von Homöopathen anerkannten Absolutheitsansprüche der Schulmedizin laufen dann natürlich darauf hinaus, dass ihr, der Schulmedizin, zugleich die selbstverständliche Berechtigung zugestanden wird, von allen anderen Medizinen Erklärungsnachweise gemäß ihres eigenen, als universal unterstellten Wirkungsmodells einfordern zu dürfen. Wenn man sich zu dieser Haltung verstehen will, dann gibt man faktisch das homöopathische Wissenschaftsparadigma auf und ordnet sich dem schulmedizinischen Paradigma ein bzw. unter. Über diese Konsequenz sollte sich ein jeder Homöopath hinreichend im Klaren sein, denn ein solcher Schritt lässt die praktische therapeutische Arbeit, die unter diesen Umständen erfolgt, keinesfalls unbeeinflusst.

\section{Das „Wir" und die Homöopathenschaft}

Wie zu sehen, handelt es sich bei dem beanstandeten Ausspruch bezüglich fehlenden Wissens, warum Homöopathie wirkt, um diejenige Art von Wissen, das mit den schulmedizinischen Vorstellungen als kongruent gedacht wird, das aber für uns, die wir uns in einem anderen Wissenschaftsparadigma bewegen, nur eine untergeordnete Bedeutung besitzt. Deshalb braucht uns diese Kritik auch nicht vordringlich zu interessieren, noch besteht irgendeine ersichtliche Notwendigkeit, uns von diesen paradigmenfremden Anforderungen irritieren lassen zu müssen oder sie gar zu übernehmen.

Nachdem dieser Sachverhalt, welches Wissen angeblich fehlt, soweit geklärt ist, wenden wir uns nunmehr dem Subjekt des betreffenden Satzes zu, dem „wir“ im „wir wissen nicht, warum unsere Arzneien wirken“. Das Personalpronomen „wir“ bezeichnet in grammatischer Hinsicht üblicherweise etwas Verbindendes. Es zeichnet Menschen aus, die, aus welchen Umständen und mit welchen Intentionen auch immer, sei es freiwillig oder unfreiwillig, eine Art von Gemeinschaft bilden.

Im vorliegenden Sprachgebrauch verhält sich dies jedoch, wie unschwer zu erkennen ist, anders. Dieses „wir“, das in der von mir aufgegriffenen Aussage verwendet wird, konstituiert nun gerade keine Gemeinsamkeit, auf welche sie semantisch doch abzuheben scheint, sondern bricht eine solche auf, oder, um es genauer auszudrücken: Das Personalpronomen „wir“ zeigt in seiner hier ungerechtfertigten Verwendung, dass genau eben dort ein fundamentaler Bruch in der Homöopathenschaft vorliegt, wo durch die plurale Ausdrucksweise eine Gemeinsamkeit supponiert wird bzw. werden soll, die in
Wirklichkeit nicht besteht, so wünschenswert ihre Existenz in der Tat wäre.

Denn es ist nicht zu übersehen, dass genau hier derzeit eine markante Trennlinie innerhalb der Homöopathie verläuft, eine Trennlinie zwischen denjenigen, die auf der Basis unseres Paradigmas sehr genau wissen, weshalb unsere Arzneien wirken, und denjenigen, die von großer Unsicherheit angekränkelt sind, da sie meinen, sie dürften ihrem ureigenen (homöopathischen) Wissen nicht vertrauen, so lange es nicht als durch naturwissenschaftlich approbierte Methodik abgesichert erscheint.

\section{Die Schwäche der Homöopathie ...}

Man kommt, sieht man sich diese Bestandsaufnahme an, nicht um die Feststellung herum, dass die Homöopathie tatsächlich unter einer nicht unerheblichen Schwäche leidet. Doch diese Schwäche der Homöopathie ist von ganz anderer Natur, als ihre schulmedizinischen Kritiker und die durch deren permanente Vorstöße verunsicherten Homöopathen denken.

Die Homöopathie zeigt sich in mancher Hinsicht schwach, aber nicht deshalb, weil sie bestimmten schulmedizinischen oder naturwissenschaftlichen Wissenschaftskriterien nicht genügen kann - was so auch gar nicht möglich wäre, ohne sich selbst aufzugeben -, sondern weil bei etlichen Homöopathen das Wissen um den wissenschaftlichen Charakter ihres eigenen Paradigmas noch immer ungenügend ausgeprägt ist und dementsprechend das Selbstbewusstsein, Vertreter einer wissenschaftlichen Medizin zu sein, erhebliche Lücken aufweist - Lücken, in welche die schulmedizinisch orientierte Kritik mit großer Freude und leider auch mit immer noch unverhältnismäßig großem Erfolg hineinzustoßen vermag.

Diesem Missstand eines nicht adäquat verbreiteten Selbstbewusstseins, die Wissenschaftlichkeit unserer Medizin betreffend, lässt sich jedoch wirksam begegnen.

\section{... und was man dagegen tun kann}

Die Lösung des Problems ist denkbar einfach und wartet keineswegs mit Neuigkeiten auf. Alles Wissen, das dazu erforderlich ist, ist längst ausgearbeitet, liegt parat und muss nur noch aufgegriffen und sinnvoll eingesetzt werden.

Der erste Schritt besteht darin, sich in aller Klarheit das homöopathische Wissenschaftsmodell als vollständiges, kohärentes und ausreichend gut in sich begründetes Paradigma zu vergegenwärtigen, das in dieser Form mit dem Paradigma der konventionellen Medizin nicht kommensurabel ist. 
In einem zweiten Schritt gilt es sich klar zu machen, dass kein Paradigma exklusive oder absolute Geltungsansprüche gegenüber einem anderen Paradigma in wissenschaftstheoretisch begründbarer Weise erheben kann. Wo dies versucht wird, verlässt der jeweilige Akteur den Boden wissenschaftsfähiger Argumentation. Und der andere, in unserem Falle die Homöopathie, ist nicht gehalten, dem Kritiker bei der Erhebung solcher absoluter Ansprüche Glauben zu schenken, sondern sollte fortfahren, seine eigenen Geltungsansprüche auch weiterhin ernst zu nehmen und sie konsequent aus seinem eigenen Paradigma zu begründen. Auf diese Weise kann die Homöopathie auch die Kriterien zur Beurteilung der Wirksamkeit ihrer Arzneien, also die Beantwortung der Warum-Frage, um die es uns in dieser Abhandlung zu tun ist, wissenschaftlich überzeugend aus sich heraus entwickeln.

Sind diese beiden genannten Schritte erfolgt, also erstens die Vergegenwärtigung, dass die homöopathische Medizin über ein eigenständiges Wissenschaftsparadigma verfügt, und zweitens, dass keine wissenschaftliche Position vorstellbar ist, die diesem Paradigma gegenüber mit guten Gründen Superioritätsansprüche erheben kann, dann besteht der dritte Schritt als praktischer Schritt darin, diesem Wissen ausreichend Präsenz in der Selbstwahrnehmung der Homöopathenschaft zu verschaffen. Denn es ist genau dieses Wissen, das die Vertreter des wissenschaftlichen Paradigmas der Homöopathie miteinander teilen müssen, um unsere Disziplin als eigenständige zu erhalten und die erfolgreiche Arbeit im Geiste Hahnemanns fortsetzen zu können.

\section{Selbstverständnis als Homöopathen}

Eine wichtige Maßnahme zur Erreichung dieses Ziels könnte darin bestehen, dieses Wissen um die wissenschaftstheoretische Situierung unseres Paradigmas als Grundlagenwissen in die Ausbildungsprogramme von angehenden homöopathischen Medizinern - handele es sich um Ärzte oder Heilpraktiker - aufzunehmen und es dadurch in ihrem Selbstverständnis als Homöopathen zu verankern. Möglichst früh sollten die Adepten unserer Medizin zu begreifen lernen, auf welchen wissenschaftlichen Grundlagen sie sich in ihrem Denken und bei ihrer praktischen Arbeit bewegen. Das fördert nicht nur die notwendige Konsolidierung der Homöopathenschaft nach innen, sondern stärkt sie auch für die Aufgabe der Selbstbehauptung unseres Paradigmas nach außen.

Aufgrund des exakteren Verständnisses der paradigmatischen Differenz zwischen Homöopathie und Schulmedizin würden die Homöopathen somit besser instand gesetzt, den Wissenschaftscharakter unserer Medizin gegenüber unhaltbaren Vorwürfen angeblicher Unwissenschaftlichkeit mit wissenschaftstheoretisch elaborierten Argumenten zu vertreten, sowie unhaltbare Superioritätsansprüche, mit denen wir vonseiten der konventionellen Medizin noch immer konfrontiert werden, mit hieb- und stichfesten Argumenten zurückzuweisen.

Gelingt die Bewältigung dieser Aufgabe - und dies stellt nicht weniger dar als die Zukunftsaufgabe für die Homöopathie überhaupt -, dann könnte der derzeit noch immer nicht überwundene Zustand der wissenschaftlichen Selbstzweifel und Irritierbarkeiten innerhalb der Homöopathie, den ideologisch motivierte Angriffe von außen stets aufs Neue zu befeuern verstehen, hoffentlich irgendwann der Vergangenheit angehören.

\section{Über den Autor}

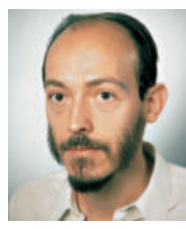

\section{Wolfgang Würger}

Dr. phil. Beschäftigung mit dem Krebsphänomen seit Mitte der 1980er-Jahre. Seit 1997 als Heilpraktiker in eigener Praxis mit Behandlungsschwerpunkt Onkologie. Forschungen und Publikationen in Philosophie, Medizin und Wissenschaftstheorie.

Korrespondenzadresse
Dr. phil. Wolfgang Würger
Prinz-Eugen-Str. 1
79102 Freiburg
E-Mail: info@praxis-dr-wuerger.de

Bibliografie

DOI https://doi.org/10.1055/a-0638-8814

AHZ 2018; 263: 4-9

(c) Georg Thieme Verlag KG Stuttgart. New York

ISSN1438-2563 